\title{
Le Città Invisibili de Italo Calvino en el contexto de la arquitectura visionaria italiana de los 60/70: Archizoom y Superstudio.
}

\author{
Italo Calvino's Le Citta Invisibili within the \\ context of the italian visionary architecture on \\ the sixties and seventies decades: Archizoom and \\ Superstudio.
}

Iván MOURE PAZOS

Istituto di Studi Superiori (Universidad de Bolonia)

y Fundación Pedro Barrié de la Maza

RESUMEN: El presente artículo pretende arrojar luz sobre la relación existente entre Le cittá invisibili de Italo Calvino y la arquitectura utópica de los años 60 y 70 en Italia, principalmente aglutinada en torno a los grupos florentinos Archizoom y Superstudio. El principal atractivo de esta investigación estriba en la exposición de una novedosa hipótesis: dicha obra no ha sido precursora de ningún movimiento visionario -como usualmente se cree- sino que ésta se inserta dentro de un debate preexistente, -fuertemente politizado- sobre la concepción de la ciudad existente en la Italia del momento. La originalidad de la obra de Italo Calvino, reside en abordar dicho debate desde el punto de vista literario y no arquitectónico pero de ningún modo constituyó el inicio de un nuevo movimiento arquitectónico, artístico o urbano. Dada esta peculiaridad, surgirá uno de los casos contemporáneos más interesantes de diálogo interdisciplinar ecfrástico entre arquitectura y literatura.

Palabras clave: Italo Calvino, Le città invisibili, Archizoom, Superstudio.

ABSTRACT: The paper tries to clarify the connection between Italo Calvino's Le cittá invisibili and the Florence design studios Archizoom and Superstudio which mainly constituted the utopian architecture movement during 1960s and 1970s. The novelty of this research approach lies in presenting Le cittá invisibili not as precursor of any visionary movement, as it is commonly believed, but just the opposite. Therefore, this book must be inserted within the previously existing context of a strong political debate held in Italy about the notion of city. The original approach of Le cittá invisibili is the fact it participates in the aforementioned debate from a literary point of view, but not architectonic; and, in no way, it constitutes the creation of a new artistic or urban architectural movement. Due to this particularity, one of the most interesting contemporary cases of ekphrastic interdisciplinary dialogue between architecture and literature will originate.

Key words: Italo Calvino, Le città invisibili, Archizoom, Superstudio. 
"Possiamo distinguere due tipi di processi immaginativi: quello che parte dalla parola e arriva all'immagine visiva e quello che parte dall'immagine visiva e arriva all'espressione verbale".

Italo Calvino. Lezioni Americane (1985)

A mi madre

\section{LE CITTÀ INVISIBILI COMO CRÍTI- CA URBANA}

Con motivo del cuarenta aniversario de Le città invisibili (1972) de Italo Calvino me dispongo a exponer mis tesis sobre lo que, tras meses de investigación, he concluido sobre la relación de dicha obra con el mundo de la arquitectura visionaria de los años 60 y 70 en Italia ${ }^{1}$. Para ello, será indispensable, primeramente, detenernos en el verdadero significado de la obra, a fin de esclarecer, en la medida de lo posible, sus pretensiones y alcance arquitectónico ${ }^{2}$. Durante cuarenta años, cientos de artículos han cimentado el corpus crítico de Le città invisibili desde diferentes disciplinas humanísticas y científicas:

“Músicólogos, arquitectos y urbanistas, historiadores del arte, geógrafos y filósofos posmodernos, han invadido este pequeño librito paseándose por sus deliciosas hojas, cual exploradores, en busca de datos y explicaciones que ayudaran a una mejor comprensión de la obra calviniana"3.

\footnotetext{
${ }^{1}$ El presente artículo se encuadra dentro de la investigación postdoctoral financiada por la Fundación Pedro Barrié de la Maza "Le cittá invisibili de Italo Calvino y la arquitectura utópica" que, bajo la supervisión de R. MILANI, forma parte del Laboratorio di Ricerca sulle Città, (Istituto di Studi Superiori. Università di Bologna). Proyecto internacional que, desde un prisma totalmente interdisciplinar, aborda el tema de la ciudad, constituido por diferentes académicos internacionales, entre los que se encuentran afamados especialistas como U. ECO o D. VILLANUEVA PRIETO.

2 Teniendo en cuenta la pasión que ha suscitado la obra de Italo Calvino fuera de Italia, -en especial en el ámbito hispanoamericano- me remito a citar los textos en el idioma original en el que fueron escritos.

${ }^{3}$ I. MOURE PAZOS, "De la ciudad visible a Le città invisibili de Italo Calvino: Utopía y urbanismo", Asociación Aragonesa de Criticos de Arte, nº 18, enero-marzo
}

De entre todos estos diálogos interartísticos, el más fructífero quizás sea aquel que considera la obra como referente arquitectónico y urbanístico. Sin ánimo de resultar tedioso, subrayo en nota al pie, las páginas de libros fundamentales para entender esta compleja relación; es decir, aquellas páginas donde se hace patente el vínculo entre lo leíble y lo visible, entre Le città invisibili y la arquitectura. Se trata de estudios cruciales para comprender esta compleja traslación, o si se quiere, diálogo interdisciplinar, al que haremos alusión continuada a lo largo de la investigación, y que, actualmente, constituyen un verdadero estado de la cuestión ${ }^{4}$.

2012, consultado el 3 de octubre de 2013. URL:file:///C:/ Users/TECNO\%20BLU/Downloads/Dialnet-DeLaCiudadVisibleALeCittaInvisibiliDeItaloCalvino-3927747. pdf. Sobre ello apunta el propio escritor I. CALVINO, "Italo Calvino on Invisible Cities", Columbia, no 8, 1983, p. 42.: "[...] Ci sono critici psicoanalitici che hanno trovato le radici profonde del libro nelle evocazioni veneziane di Marco Polo, come un ritorno ai primi archetipi della memoria; mentre studiosi di semiologia strutturale hanno detto che è nel punto esattamente centrale del libro che bisogna cercare: e hanno trovato un' immagine di assenza, la città chiamata Bauci. Qui è chiaro che il parere dell'autore è di troppo: il libro, come ho spiegato, si è fatto un po'da sé, ed è solo il testo come che può autorizzare o escludere questa o quella lettura".

${ }^{4}$ Por orden alfabético resaltamos las siguientes páginas de estudios críticos (no se incluyen las fuentes primarias de Italo Calvino; sus propios escritos): F. AÍNSA, "Las ciudades invisibles de Italo Calvino. ¿Utopía visionaria o crónica de la memoria del futuro?", en Borges, Calvino, la literatura, (Vol. II), Madrid, 1996, pp. 61-85; M. BARENGHI, “Gli abbozzi dell'indice: Quattro fogli dall' archivo di Calvino" en M. BARENGHI, G. CANOVA, B. FALCETTO (dirs.), La visione dell 'invisibile. Saggie materiali sur Le città invisibili di Italo Calvino, Milán, 2002, pp. 74-95; M.C. BARRADO BELMAR, “Marco Polo- Italo Calvino: semiótica de las ciudades en Le città invisibili", Actas del IV simposio internacional, Sevilla, Asociación española de semiótica, Sevilla, 3-5 de diciembre de 1992, pp. 543- 553; ÍDEM, "Semiótica de las ciudades en Le città invisibili de Italo Calvino", Actas del VI congreso 
nacional de italianistas, Madrid, 1994, pp. 71- 80; ÍDEM, "Gematría y poética en Le città sottili de Le città invisibili de Italo Calvino", Cuadernos de filología italiana, no 16, 2009, pp. 275-285; F. BIAMONTI, “Un ligure cosmopolita", en G. BERTONE (dir.), Italo Calvino, la letteratura, la scienza, la città, Atti del convegno nazionale di studi di Sanremo, Génova, 1988, pp. 67-97; P. BRAFFORT, “Italo Calvino sur les sentiers du labyrinthe", Magazine littéraire, n 398, 2002, pp. 57-60; G. DEMATEIS, “La superficie e l'altrove. Dal Marco polo di Italo Calvino al linguaggio cose nella geografia d'oggi", en G. BERTONE (dir.), Op. cit., pp. 94-99; N. FEAL, "La ficcionalización del territorio", Bifurcaciones: revista de estudios culturales y urbanos, $\mathrm{n}^{\mathrm{o}}$ 4, 2005, consultado el 19 de octubre de 2013. URL: http://www.bifurcaciones.cl/004/Feal.htm; A. FERLENGA, "Invisible Profundities", en M. BARENGHI, G. CANOVA, B. FALCETTO (dir.), Op. cit., pp. 140-48; M. I. GARCÍA CANAL, "Las ciudades invisibles: la máquina de escribir de Italo Calvino", Relaciones, vol. 27, no 106, 2006, pp. 135-50; F. JURADO, "La ciudad invisible de los clásicos. Entre Aulo Gelio e Italo Calvino", Nova Tellus, vol. 28, no 1, 2010, pp. 271-300; P. KUON, “Critica e progetto dell'utopia: Le città invisibili di Italo Calvino" en M. BARENGHI, G. CANOVA, B. FALCETTO (dir.), Op. cit., pp. 24-41; M. LABRAÑA, "Las ciudades de Calvino: un inventario imposible", Revista laboratorio, no 0 , 2009, consultado el 20 de octubre de 2013. URL: http://www.revistalaboratorio.cl/2009/04/las-ciudadesde-calvino-un-inventario-imposible/; R. LUDOVICO, Le città invisibili di Italo Calvino: le ragioni della scrittor, Montreal, 1997, pp. 1-91; G. MANGANELLI, "Recensione inedita" (relativo a un intervento radiofonico de 1972), en M. BARENGHI, G. CANOVA, B. FALCETTO (dir.), Op. cit., pp. 105-29; M.L. MCLAUGHLIN, "Le città visibili di Calvino" en Ibídem, pp. 42-61; A.A. MEJÍA GONZÁLEZ, Sexta propuesta literaria de Italo Calvino, "Consistencia": un acercamiento a través de Las ciudades invisibles, Morelia, 2011, pp. 52-74; L. MODENA, Italo Calvino's Arquitecture of Lightness. The Utopian imagination in an Age of Urban Crisis, New York, 2011, pp. 1-264; ÍDEM, "Mi veniva da Scrivere città sottili come le sue sculture": La scultura di Fausto Melotti nelle Città invisibili di Italo Calvino, Letteratura y arte, ํํ2, 2004, pp. 217-242; C. OSSOLA, "L' invisibile e il suo "dove": "geografía interiore" di Italo Calvino", Lettere Italiane, vol. 39, no 2, 1987, pp. 242-48; R. PIERANTONI, "Metafore di un mappa", en G. BERTONE (dir.), Op. cit., pp. 89-93; J.C. ROVIRA, “Borges, Calvino y la fundación literaria de ciudades", en Borges, Calvino, la literatura..., pp. 8597; E. SÁNCHEZ GARAY, "Las ciudades invisibles y deseadas", Universidad Michoacana de San Nicolás de Hidalgo, s/no ${ }^{\circ}, 2002$, consultado el 1 de noviembre de 2013. URL: http://dieumsnh.qfb.umich.mx/ciudades_invisibles.htm; M. VAN MONTFRANS, “L'enchâssement des énigmes. Les Villes invisibles de Calvino dans La vie mode d'emploi de Perec", en C. REGGIANI y B. MAGNÉ (dir.), Écrire l'énigme, Paris, 2007, pp. 115-26.
Como bien apunta Gombrich: nunca debe formularse una hipótesis sobre una obra sí el autor ya ha explicado la suya ${ }^{5}$. Se trata de supeditar la literatura crítica -o fuentes secundarias- sobre Le città invisibili a las fuentes primarias proporcionadas por el propio autor para así comprender mejor su verdadero significado. Afortunadamente, Italo Calvino fue un escritor prolífico y nos ha dejado multitud de apuntes sobre Le città invisibili que, de una manera más que clarividente, se nos ofrece como un excepcional viático $\mathrm{u}$ hoja de ruta indispensable para descifrar el alcance de esta obra que se encuentra a medio camino entre el ensayo poético y el tratado arquitectónico.

La obra pudiera ser concebida como un mero ejercicio literario fuertemente poetizado, cuya finalidad última estribaría en una mera fantasía escritural; es decir, un mero ejercicio estético, desprovisto de todo contenido crítico, social y politizante. Sin embargo, tras esta lectura idílica y preciosista se esconde una de las críticas sociales y urbanas más acertadas y con mayor alcance surgidas en la Italia de los 60 y $70^{6}$. No podemos olvidar que Le città invisibili ha sido reseñada por algunos estudiosos como la obra más impor-

${ }^{5}$ E. GOMBRICH, Imágenes simbólicas. Estudios sobre el arte del renacimiento, II, Madrid, 2001, pp. 5-7.

${ }^{6}$ Esta hipótesis social ha sido tratada, de manera directa o indirectamente, por los siguientes autores. Por orden alfabético reseñamos las siguientes páginas de libros: F. AÍNSA, Op. cit., p. 81; M.C. BARRADO BELMAR, "Gematría y poética...", pp. 275-285; G. BONURA, Invito alla lettura di Calvino, Milano, 1972; M.L. MCLAUGHLIN, Op. cit., p. 42; M.I. GARCÍA CANAL, Op. cit., p. 147; C. MATEOS MARTÍN, "La comunicación poética y política de la ciudad: Análisis de la ciudad como argumento de información política electoral, frente a Las ciudades invisibles de Italo Calvino", Espéculo, no 23, 2003, consultado el 15 de noviembre de 2013. URL: https://pendientedemigracion.ucm.es/info/especulo/numero23/ciudades.html; A. MEJÍA GONZÁLEZ, Op. cit., pp. 9, 47; E. MONDELLO, Italo Calvino, Pordenone, 2000, p. 110; U. MUSARRA-SCHROEDER, Il laberinto e la rete: percorsi moderni e postmoderni nell'opera di Italo Calvino, Roma, 1996, pp. 83-97; R. PIERANTONI, Op. cit., p. 90; J.C. ROVIRA, Op. cit., pp. 85-86; E. SÁNCHEZ GARAY, Op. Cit. ; M. VAN MONTFRANS, Op. cit., p. 117. 
tante de Italo Calvino, convirtiéndose en un auténtico best-seller de la narrativa contemporánea europea ${ }^{7}$.

Le città invisibili no sólo es una reflexión crítica de denuncia social, sino también un excepcional testimonio autobiográfico de la relación de Italo Calvino con el entorno que le circunda:

“La incógnita que el autor nos plantea en este librito, surge de dos grandes preguntas entrelazadas: qué es la ciudad moderna y cuál nuestra relación con ella [...] Le città invisibili es un canto a la ciudad ideal en un momento en que la urbe, la megalópolis, se gigantiza para empequeñecer al hombre. El significado de ciudad comienza a perderse, porque ésta -como entidad urbana independiente- ya no se supedita a las verdaderas necesidades de las personas, sino viceversa. Como alternativa a este espacio infernal, el autor propone una ciudad utópica e imposible: le città invisibili. Ésta, es la ciudad ideal que nunca encontramos, pero que necesitamos irremediablemente buscar para alimentar nuestros verdaderos sueños. [ ] La ciudad visible, por consiguiente, es inasible y, en su dimensión compleja, no puede aprehenderse, por lo tanto, no existe como tal. La no existencia genera una necesidad de búsqueda, pero ésta ha de desarrollarse en base a una exploración individual de la propia espacialidad, tan ecléctica y poliédrica que, siguiendo un principio de invisibilidad, no admite ningún tipo de canon urbano o escuela arquitectónica. Se trata de las ciudades que comportan el itinerario vital de Italo Calvino; una urbe utópica, alejada de la ciudad homogenizada, turistizada y globalizada, donde el único modo de conducir-

\footnotetext{
${ }^{7}$ Sobre ello inciden: M. BELLO VALDÉS, "Italo Calvino: el estupor de estar en el mundo", Palabras poetas e imágenes de Italia, Méjico, 1997, p. 38; G. MANGANELLI, Op. cit., p. 105; M.L. MCLAUGHLIN, Op. cit., p. 59.
}

se a ella pasa por un viaje discontinuo, donde resultaría del todo infructuoso establecer una ruta en el mapa, o fijar una fecha de llegada [...] De ahí, el precisar una ciudad sintiente y humana en sintonía a nuestros anhelos, pasiones y urgencias más intrínsecas. La ciudad utópica es, entonces, una "alegoría del deseo" que nace tanto de nuestra inadaptación al medio como de la necesidad de una búsqueda espacial satisfactoria; un laberinto interior donde se especula con los valores meramente territoriales y urbanos a través de la propia imaginación" ${ }^{\prime 8}$.

Con acierto, Mario Barenghi definirá al autor como "[...] scrittore dalla vocazione eminentemente urbana" Esto probablemente por las constantes y reiteradas alusiones que Italo Calvino realiza en torno a la problemática de la ciudad y su habitabilidad en el conjunto de su obra literaria y epistolar. Entre 1957 y 1985 -año de su muerte- contamos con numerosos documentos explicativos sobre esta querencia sobre el hecho, la reflexión y la crítica urbana $^{10}$. Una de las fuentes fundamentales

${ }^{8}$ I. MOURE PAZOS, Op. cit.

${ }^{9}$ M. BARENGHI, Op. cit., p. 94.

${ }^{10}$ Por orden alfabético resaltamos las siguientes páginas de cartas y escritos de Italo Calvino. Estas son las fuentes directas en las que el autor aborda la problemática de la ciudad:

LETTERE: I. CALVINO, (1973), “Lettere a Angel Rama (Caracas)”, en L. BARANELLI (ed.), Calvino, Lettere, Milán, 2001, p. 1222; I. CALVINO (1974), “Lettere a Caterina de Caprio (Napoli)", Ibídem, pp. 1234-1235; I. CALVINO (1973), "Lettere a Claudio Varese (Firenze)", Ibídem, pp. 1192-1194; I. CALVINO (1983), "Lettere a Francesco Tentori (Roma)", Ibídem, p. 1500; I. CALVINO (1969), “Lettere a Franco Maria Ricci”, Ibídem, pp. 10591060; I. CALVINO (1973), “Lettere a Geno Pampaloni (Firenze)", Ibídem, p. 1202; I. CALVINO (1972), "Lettere a Geno Pampaloni (Firenze)", Ibídem, pp. 1184-1185; I. CALVINO, (1972), "Lettere a Gianni Celati (Bologna)", Ibídem, p. 1150; I. CALVINO (1974), "Lettere a Giovanni Falaschi (Firenze)", Ibídem, pp. 1257-1258; I. CALVINO (1977), "Lettere a Guido Calogero (Roma)", Ibídem, pp. 1341-1342; I. CALVINO (1970), "Lettere a Pietro Citati. La Castellacia, Guiuncarico (Grosseto)", Ibídem, p. 1089; I. CALVINO (1976), "Lettere a Sandro Briosi (Gro- 
para comprender esta obsesión geográfica es la primera carta que Italo Calvino envía al célebre editor Franco Maria Ricci, -tres años antes de la publicación de Le città invisibili-: "Per lunghi anni soffersi d'una nevrosi geografica: non riuscivo a stare tre giorni di seguito in nessuna città o luogo"11, secundada cinco años después por una segunda epístola al célebre editor parmesano -escrita en francés- donde vuelve a arremeter nuevamente sobre este particular ${ }^{12}$.

Sendas epistolas a Franco Maria Ricci adelantan algunas de las explicaciones indispensables del autor sobre Le città invisibili publicadas posteriormente en diferentes libros de ensayos en las que pretende aclararnos qué objetivo perseguía la obra y su significado preciso:

"Questa idea, che percorre tutto il mio libro Le città invisibili, mi viene dal modo di vivere che è ormai di molti di noi: un continuo passare da un aeroporto all'altro, per fare una vita pressoché uguale in qualsiasi città ci si trovi"13. "[...] Forse stiamo avincinandoci a un momento di crisi della vita urbana [...] Le città invisibili sono un sogno che nasce del cuore delle città

ningen)", Ibídem, p. 1304; I. CALVINO (1972), "Lettere a Vittorio Spinazzola", Ibídem, p. 1186; I. CALVINO (1974), "Una lettera en due versioni”, en Eremita a Parigi, Milán, 1994, pp. 181-182.

SAGGI e ROMANZO: I. CALVINO (1974), “Eremita a Parigi" en Eremita a Parigi..., pp. 190-207; I. CALVINO "Italo Calvino on Invisible Cities", Columbia, nº 8, 1983, pp. 37-42; I. CALVINO (1985), “Intervista di Maria Corti", en Eremita a Parigi..., pp. 271-281; I. CALVINO (1953), La speculazione edilizia, Milán, 2000; I. CALVINO (1963), La giornata d'uno scrutatore, Milán, 1995; (Sendas obras han de ser apreciadas en su totalidad por ser consideradas por M. L. MCLAUGHLIN como precedentes directos de Le città invisibili. Vid. M.L. MCLAUGHLIN, Op. cit., p. 42), I. CALVINO (1972), Le città ivisibili, Milán, 1993.

${ }^{11}$ I. CALVINO (1969), "Lettere a Franco Maria Ricci”, en L. BARANELLI (ed.), Op.cit., p. 1059.

${ }^{12}$ I. CALVINO (1974), “Una lettera en due versioni”, en Eremita a Parigi..., pp. 181-182.

${ }^{13}$ I. CALVINO (1974), "Eremita a Parigi", Ibídem, p.192. invivible [...] Credo che non sia solo un'idea atemporale di città quello che il libro evoca, ma che vi si svolga, ora implicita ora esplicita, una discussione sulla città moderna"14.

Quizás, todo ello con el fin de "[...] cercare e saper riconoscere chi e cosa, in mezzo all'inferno, non è inferno, e farlo durare, e dargli spazio" ${ }^{\prime 15}$.

\section{LE CITTÀ INVISIBILI Y SUS PRECE- DENTES VISIONARIOS}

Le città invisibili, pese a pertenecer oficialmente al ámbito de la literatura, participa activamente y, de una manera claramente intencional, del debate arquitectónico europeo -esencialmente italiano- de los años 60 y 70 liderado por los movimiento visionarios de Archizoom y Superstudio; de hecho, actualmente, esta lectura se antoja indispensable para comprender en su totalidad la obra de Italo Calvino a tratar ${ }^{16}$. No es baladí que Le città invisibili sea una obra italiana, así como tampoco que la principal reflexión sobre el hecho utópico y urbano estuviera teniendo lugar en la convulsa Italia del momento:

“En Italia, el escenario político estaba caracterizado por luchas que marcaban entrecruzamientos de diversos factores, tales como: el milagro económico de pos-guerra y el fuerte

${ }^{14}$ I. CALVINO, “Italo Calvino on Invisible...", p. 41.

${ }^{15}$ I. CALVINO (1972), Le città ivisibili,..., p. 164.

${ }^{16}$ Amén de influencias explícitamente visionarias, parece bastante plausible que Italo Calvino conociera algunos escritos teóricos de arquitectura que agitaron y abrieron el debate urbano de la época, probablemente el libro de A. ROSSI (1966), L'architettura della città, Macerata, 2011 -digno heredero de The death and life of great american cities, New York, 1993, de J. JACOPS (1961), o Complexity and contradiction in architecture, New York, 1966, de R. VENTURI- así como las incisivas investigaciones de M. TAFURI, entre las cuales destacaré -en sucinto prontuario-: "Per una critica dell'ideologia architettonica", Contropiano, Materiali Marxisti, no 1, 1969, pp. 31-79, y "Design and technological utopia" en E. AMBASZ (ed.), The New Domestic Landscape, Achievements and Problems of Italian Design, New York, 1972, pp. 308-404. 
ritmo de industrialización, conducido por un gobierno de centro-derecha formado básicamente por una coalición entre el Partido Demócrata Cristiano [...] y el Partido Socialista Italiano; los procesos de suburbanización, que deberían absorber el éxodo poblacional en dirección a las área metropolitanas, principalmente la población venida del sur (agrario) al norte (industrial); la insurgencia de movimientos de contestación estudiantil [...] Todas estas cuestiones contribuirán a la formulación de un espíritu crítico en relación a la realidad profesional que influenció la generación de Archizoom y Superstudio"17. "La crítica a la sociedad, el capitalismo y sus instituciones llevó a los dos grupos hacia una reflexión profunda sobre las propias estructuras de la disciplina arquitectónica, ya fuera sobre el oficio de arquitecto o de diseñador. Así, Archizoom y Superstudio decidirán atacar la arquitectura utilizando sus propias herramientas disciplinares [...] denunciando un mundo completamente colonizado por la instrumentalización de la técnica y el dominio del capital" $^{\prime 18}$.

Este contexto no será ajeno a las propias inquietudes de Italo Calvino, afín, como sabemos, a la crítica capitalista, y por ende, firme detractor de la voraz especulación urbana acaecida en aquellos años. Enlazando con el primer epígrafe de este artículo, no estará de más recordar que, tanto el escritor como sus "colegas" visionarios, se gestaron al amparo de los movimientos de izquierdas, dotando a sus propuestas arquitectónicas y urbanas de un fuerte contenido politizante y social. Por lo tanto, no debemos pasar por

${ }^{17}$ R. KAMIMURA, Tecnología, emancipaçao e consumo na arquitectura dos anos sessenta: Constant, Archigram, Archizoom y Superstudio, (Tesis Doctoral), Sao Paulo, 2003, p. 154. (Traducción del Portugués al Castellano realizada por el autor del artículo).

${ }^{18}$ Ibídem, p. 149. alto que Le città invisibili y los movimientos visionarios italianos precedentes, se desarrollen en un mismo plano artístico -fuertemente influenciado por la crítica política y social- que, en definitiva, postulaban una alternativa a la ciudad fagocitante y especulativa capitalista.

Sobre este aspecto vuelve Italo Calvino en una carta escrita a Guido Calogero en la que incide, nuevamente, sobre la intencionalidad política de su obra. Se trata de un documento excepcional, donde el escritor no sólo aclara su posición frente los abusos especulativos de la ciudad sino que contextualiza su obra dentro de la larga tradición visionaria europea-de ahí su enorme importancia- que, como sabemos, florece en Italia con Piranesi ${ }^{19}$ y se disuelve en Italia con Le città invisibili, Archizoom y Superstudio en $1972^{20}$ :

"Poi per tutto l'Ottocento utopisti ed esteti, da Fourier a Ruskin, a Morris rifiutano la bruttezza della civiltà contemporanea che si esprime nell'urbanistica e nei prodotti d'uso, e propongo non solo un uso diverso, ma cose (e case) diverse. Questa linea continua nel nostro secolo, con la Bauhaus, con l'industrial design, etc. Si tratta certo di progetti falliti

${ }^{19}$ Ibídem, pp. 192-194. Kamimura establece una correlación entre una de las obras más afamadas de Archizoom, No Stop City (1971) y Il Campo Marzio dell'Antica Roma (1761-62) de Piranesi.

${ }^{20}$ Ibídem, p. 207. "Historiadores y críticos parecen concordar con la hipótesis de que los años sesenta terminaron en 1973. Esta es, al menos, la propuesta de Frederic Jameson, que apunta el período comprendido entre 1972 -año de la publicación de Le città invisibili- a 1974 como repleto de indicaciones significativas de esta ruptura: el golpe político en Chile, y la permanencia de casi todos los países de América latina bajo dictaduras, la retirada de la tropas de Vietnam, el desgaste y fragmentación del movimiento negro y del movimiento feminista, el acuerdo común entre el partido Comunista y el partido Socialista de Francia, la crisis de la nueva izquierda [...], la crisis del petróleo de 1973-74 [...] 1972 también es el año de la exposición Italy: the new domestic landscape en el MOMA de New York, donde, conforme observamos, Superstudio y Archizoom reconocían que su tarea intelectual había llegado a su fin. 
in partenza per la loro utopistica pretesa che il mondo cambi atraverso il cambiare della forma delle cose. Ma il cercare di leggere nelle cose il dramma degli uomini non mi sembra procedimento sbagliato. Ecco dunque che non sono soltando le istituzioni que intendevo come cose giuste o ingiuste ma proprio le cose e quella cosa per l'eccellenza che è la città. (Il mio ultimo libro Le città invisibili, 1972, verte in gran parte su questi temi). Dico insomma qualcosa di abbastanza ovvio e che Lei stesso proverà ogni volta che esce di casa: la Roma delle speculazioni edilizie porta in sé 1'impronta della somma d'ingiustizie da cui nasce, independentemente dall' uso che se ne fa o farà" ${ }^{21}$.

Al igual que la larga lista de visionarios que conforman el corpus utópico, Italo Calvino, nos propone una ciudad particularizada de acuerdo con los criterios individuales de habitabilidad. Con razón apostilla Ruggero Pierantoni: “Non si può mettere una mappa prima delle città invisibili perché la mappa è chiaramente una mappa del desiderio, della nostalgia, è una mappa interiore $^{\prime 22}$. La misma premisa perseguida y esgrimida por Archizoom y Superstudio, los cuales, cabe matizar, fueron los primeros arquitectos en tender puentes con el arte conceptual, el diseño y sobre todo con el mundo de la cinematografía y la literatura. Por esta misma razón, Le città invisibili casa tan bien con la tradición arquitectónica de los visionarios italianos, quizás porque éstos, ya desde sus inicios, fueron concebidos como una propuesta interartística permeable al diálogo de las diferentes artes, alejándose de concepciones puramente arquitecturales:

"Ya en 1966 -6 años antes de la publicación de Le città- arquitectos adscritos a Superstudio y Archizoom

${ }^{21}$ I. CALVINO, "Lettere a Guido Calogero", en L. BARANELLI (ed.), Op.cit., pp. 1341-1342.

${ }^{22}$ R. PIERANTONI, Op. cit., p. 90. como Adolfo Natalini, Piero Frasinelli, Cristiano Toraldo de Francia, Andrea Branzi, Gilberto Corretti, Paolo Deganello o Massimo Morozzi, comienzan a promulgar esa idea -tremendamente calviniana- de que la ciudad debe supeditarse al hombre así como servirle en sus necesidades más inmediatas y estéticas, puesto que, como sabemos, es el propio hombre quien la construye para su goce y disfrute. Por ello, comienzan a incidir en una serie de postulados urbanos que redundarán en un crítica a la ciudad moderna, toda vez, reivindican una nueva ciudad interior; una nueva città invisibili [...] Algunos ejemplos palmarios de este fructífero diálogo interdisciplinar entre la obra de Italo Calvino y sus predecesores utópicos pueden intuirse en las siguientes correspondencias: Octavia (ciudad telaraña) y la Maison pour Arman a Vence (1966) de Guy Rottier, Baucis e Intrapolis (1966) de Walter Jonas, Valdrada y la Macchina per le vacanze (1969) de Cristiano Toraldo di Francia, Eutropia y la ciudad móvil Continuous production conveyor belt City (1971) de Superstudio, o las ciudades sintientes de Zenobia o Rasia -entroncando con aquella "ciudad-personaje" de la que habló María Inés García- y City of the Splendid Houses (1971) propuesta por Superstudio, erigida como la ciudad más independiente, feliz y bella del mundo. Aunque quizás, sea la irónica City of order (1971) firmada nuevamente por Superstudio, la más calviniana de las enumeradas, pues, en ella, convergen todas las preocupaciones del escritor. Se trata de una ciudad congelada, inamovible y estática, de hecho, lleva 45 años gobernada por el mismo alcalde gracias al triunfo de una brillante idea: supeditar las necesidades de sus ciudadanos a la invariable City of order"23.

${ }^{23}$ I. MOURE PAZOS, "Arquitectura escrita: lecturas y relecturas sobre Le Città invisibili de Italo Calvino", Arbor, Ciencia, Pensamiento y Cultura, nº 761, 2013, p. 6. 
Una de las constantes calvinianas que másgustanalescritoresladelacontraposición de dos conceptos antagónicos: la ligereza frente a la pesantez, tomada de los viejos e indesligables postulados visionarios. Dicha contraposición será consustancial a gran parte de su producción escrita referida a la ciudad y abordada de manera especialmente teórica en su última obra inconclusa: su ciclo de conferencias para la Universidad de Harvard, posteriormente publicadas bajo rúbrica de Lezioni Americane (1985): "[...] la mia operazione è stata il più delle volte una sottrazione di peso; ho cercato di togliere peso ora alle figure umane, ora ai corpi celeste, ora alle città $[\ldots]^{\prime 24}$. Algo que se evidencia en sus obras de calado más urbano, entre las cuales, quizás, deberíamos reseñar "Quale storia laggiù attende la fine" contenido en su excepcional libro de cuentos Se una notte d'inverno un viaggiatore (1979), donde el protagonista desmaterializa toda una ciudad a su paso con la fuerza de su percepción visual hasta reducirla a su esencia minimalista. No estará de más decir que este concepto de levedad o ligereza aplicado a la desmaterialización de una ciudad opresora, burguesa y capitalista, se desarrolle de manera sincrónica a la propuesta de Superstudio, Continuous Movement (1971) de la que, con acierto, Rodrigo Kamimura dirá: “[...] las imágenes de Superstudio revelaban a primera vista una sencillez que parecía hacer que sus objetos metafísicos fluctuasen en el espacio como si estuvieran prestos a desvanecerse. De hecho, esto era lo apropiado para una arquitectura que se quería autodestructiva" 25 , con el fin de "[...] deshacerse de todos los objetos de valor y ejercitar sus capacidades lúdicas" 26 .

No ha de extrañarnos, por lo tanto, que a partir de los 70, Italo Calvino se convierta en un referente del estudio de la ar-

\footnotetext{
${ }^{24}$ I. CALVINO, Lezioni Americane, Milán, 1988, p. 5.

${ }^{25}$ R. KAMIMURA, Op. cit., p. 173.

${ }^{26}$ Ibídem., p. 177.
}

quitectura, siendo invitado por numerosas y prestigiosas instituciones de corte académico a dar ponencias sobre la problemática de la ciudad -algo a todas luces insólito por parte de un literato-. Buena muestra de este requerimiento se patentiza en la carta escrita al afamado arquitecto Francesco Tentori donde refleja su preocupación -en los últimos años de su vida y a 13 años vista de la publicación de Le cittá invisibili- por el mundo de la arquitectura y el urbanismo:

“Caro Tentori: la ringrazio molto dell'invito. Non è la prima volta che architetti e urbanisti mi sollecitano a continuare con loro e i loro allievi il discorso della Città invisibili. E non avrei proprio nulla contro; anzi $\mathrm{mi}$ piacerebbe. Ma sono stracarico di lavoro e programmare un viaggio a Venecia aposta, pero ora mi è impossibile. Il mese venturo, tra l'altro, parto per gli Stati Uniti e quest'anno non posso programmare altri viaggi $[\ldots]$ Non avevo visto gli articoli di Portoghesi e Gardella che mi segnala. Se li ha sottomano, può mandarmene uan fotocopia?"27.

Actualmente, podemos considerar a Le città invisibili (1972) de Italo Calvino como un feliz ejemplo de lo que comúnmente conocemos como ecfrásis. A nadie se le escapa ya, en el cuarenta aniversario de su publicación, que el libro se convirtió en la punta del iceberg de una revolución arquitectónica gestada a mediados de los años 60 en Italia de la mano de grupos visionarios como Archizoom y Superstudio, cuyo denominador común se centró en la subversión de los viejos y caducos postulados arquitectónicos de la burguesía del momento. Su celebrado aniversario nos brinda una excelente oportunidad para ahondar en sempiternas reflexiones sobre la ciudad: ¿Qué es la ciudad? ¿Cuál su significado?, y, sobre todo, ¿Cuál su función y deber para con nosotros?.

${ }^{27}$ I. CALVINO “Lettere a Francesco Tentori”, en L. BARANELLI (ed.), Op.cit., p. 983. 